\title{
Phase-encoded RF photonic signal generation for radar systems with a soliton crystal micro-comb source
}

\author{
Xingyuan Xu, Mengxi Tan, Jiayang Wu, Andreas Boes, Bill Corcoran, Thach G. Nguyen, Sai T. Chu, \\ Brent E. Little, Roberto Morandotti, Arnan Mitchell, and David J. Moss
}

\begin{abstract}
We demonstrate photonic RF phase encoding based on an integrated micro-comb source. By assembling single-cycle Gaussian pulse replicas using a transversal filtering structure, phase encoded waveforms can be generated by programming the weights of the wavelength channels. This approach eliminates the need for RF signal generators for RF carrier generation or arbitrary waveform generators for phase encoded signal generation. A large number of wavelengths - up to 60-were provided by the microcomb source, yielding a high pulse compression ratio of 30. Reconfigurable phase encoding rates ranging from 2 to $6 \mathrm{~Gb} / \mathrm{s}$ were achieved by adjusting the length of each phase code. This work demonstrates the significant potentials of this microcomb-based approach to achieve high-speed RF photonic phase encoding with low cost and footprint.
\end{abstract}

Index Terms-Microwave photonics, micro-ring resonators.

\section{INTRODUCTION}

Phase encoded continuous wave (CW) radio frequency (RF) signals are widely employed in low probability of intercept (LPI) radar systems, since they feature low power density and employ random codes to avoid being cracked [1-2]. In such systems, targets are resolved within range when the reflected phase encoded signals are compressed at the receiver, and a large signal bandwidth is required to achieve a high resolution [3]. Photonic techniques have significant potential to overcome the limitations of their electronic counterparts [4] due to their intrinsically broad bandwidth, immunity from electromagnetic interference, and low propagation loss [5-11].

Significant effort has been devoted to realizing photonic-assisted RF phase encoding, including approaches based on polarization modulators [12, 13], Sagnac loops [14, 15], and dual parallel modulators [16, 17]. However, these approaches all face limitations of one form or another, such as the need for complicated modulation schemes to achieve the required $\pi$-phase shifts, and the need for both high-frequency RF signal generators and arbitrary waveform generators. The former increases the complexity and instability (such as the bias drift of the modulators) of the system, while the latter greatly increase the cost, size and power consumption.

An alternative method of generating RF phase encoded signals based on transversal filtering structures has been proposed [18]. In this method, RF high-order Gaussian pulses are broadcast onto 4 wavelength channels via an intensity modulator, and then progressively delayed through a dispersive medium such that the pulse segments are assembled (concatenated) in the time domain. By controlling the phase of each pulse segment, phase-encoded waveforms can be generated. This approach reduces the need for RF sources and complicated modulation schemes, and offers a high potential phase encoding speed by tailoring the delay step and pulse width. However, it is not without challenges. The use of discrete laser arrays significantly increases the complexity and cost for further integration and limits the number of wavelengths. This in turn leads to a limited sequence length and thus a small pulse compression ratio that would limit the performance of radar systems. Secondly, the employed high-order Gaussian pulses require complicated

This work was supported by the Australian Research Council Discovery Projects Program (No. DP150104327). RM acknowledges support by the Natural

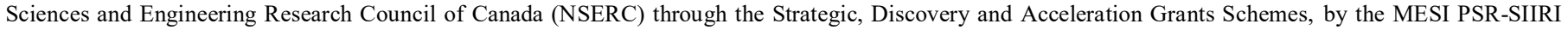

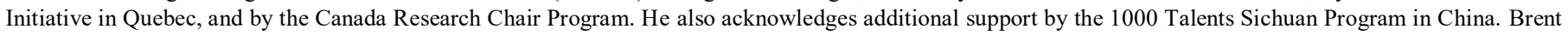
E. Little was supported by the Strategic Priority Research Program of the Chinese Academy of Sciences, Grant No. XDB24030000.

X. Xu, M. Tan, J. Wu, and D. J. Moss are with the Centre for Micro-Photonics, Swinburne University of Technology, Hawthorn, VIC 3122 , Australia. (Corresponding e-mail: dmoss@swin.edu.au).

A. Boes, T. G. Thach and A. Mitchell are with the School of Engineering, RMIT University, Melbourne, VIC 3001, Australia.

B. Corcoran is with the Department of Electrical and Computer System Engineering, Monash University, Clayton, VIC 3168 Australia.

S. T. Chu is with the Department of Physics, City University of Hong Kong, Tat Chee Avenue, Hong Kong, China.

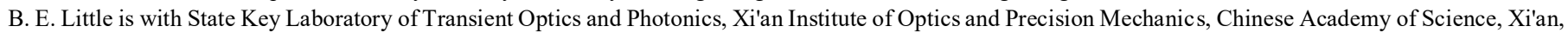
China.

R. Morandotti is with INSR-Énergie, Matériaux et Télécommunications, 1650 Boulevard Lionel-Boulet, Varennes, Québec, J3X 1S2, Canada, and adjunct with the Institute of Fundamental and Frontier Sciences, University of Electronic Science and Technology of China, Chengdu 610054, China. 
generation schemes such as high-order differentiation, which will increase the cost and complexity of the system.

Integrated microcombs [19-24], generated through optical parametric oscillation in monolithic microring resonators (MRRs), are promising multi-wavelength sources that offer many advantages including a much higher number of wavelengths and greatly reduced footprint and complexity. Thus, a wide range of RF applications have been demonstrated based on micro-combs, such as transversal filters [25-27], differentiators [28], optical true time delays [29, 30] and channelizers [31, 32], Hilbert transformers [33-35], microwave mixers [36], microwave generation [37], and more [38-43].

In this paper, we demonstrate phase encoded CW RF signal generation using an integrated soliton crystal microcomb source. The soliton crystal is a recently discovered coherent and stable solution of the Lugiato-Lefever equation $[44,45]$, which governs the nonlinear dynamics of the optical parametric oscillator. The soliton crystal stably forms through the background wave generated by a mode crossing, and features over thirty times higher intra-cavity power than traditional dissipative Kerr solitons. Thus, they can remove thermal effects and enable adiabatic pump sweeping for coherent comb generation [46, 47]. These properties, together with the broadband comb spectrum, make soliton crystals promising for demanding integrated RF photonic systems, such as the photonic RF phase-encoder introduced in this work.

Here, by multicasting an RF single-cycle pulse (instead of the high-order pulse used in [18]) onto a spectrally flattened micro-comb and progressively delaying the replicas with a dispersive medium, the RF pulse replicas are assembled arbitrarily in time according to the designed binary phase codes. The large number of wavelengths offered by the microcomb enabled 30 bits, representing an enhancement of over 4 times compared to previous work [18], for the phased encoded RF sequence, leading to a pulse compression ratio as high as 29.6. A reconfigurable phase encoding rate ranging from 1.98 to $5.95 \mathrm{~Gb} / \mathrm{s}$ was also achieved by varying the length of each phase code. These results verify the strong potential of our approach for the realization of photonic RF phase encoding schemes and represent a solid step towards the miniaturization of fully integrated radar transmitters with greatly reduced cost and footprint.

\section{THEORY}

Figure 1 illustrates the operation principle of a photonic phase encoder. First, an RF Gaussian pulse $f(t)$ with a duration of $\Delta t$ is generated. Then a discrete convolution operation between the RF pulse and flattened microcomb spectrum (denoted by a discrete signal $g[n]$ with length $N$ and binary values of 1 or -1 ) is performed with a delay step of $\Delta t$, and can be described as:

$$
(f * g)[n]=\sum_{i=1}^{N} f[n-i \cdot \Delta t] \cdot g[i]
$$

The discrete convolution operation between the RF pulse $f(t)$ and the flattened microcomb spectrum is achieved using the experimental setup in Figure 2. The RF pulse is first multi-cast onto the wavelength channels to yield replicas, which are then delayed with a progressive step that matches the pulse duration $\Delta t$. By flipping the sign of the delayed replicas according to designed phase codes $g[n]$, a phase-encoded sequence can be assembled in the time domain. The sign reversal is accomplished by spatially separating the intended positive pulses from the negative ones using the programmable wavelength dependent spatial routing capability of a waveshaper, and then directing the two separate outputs to the positive and negative inputs of a differential photodetector, respectively. The total number of RF pulse replicas $N$ is equal to the number of wavelength channels, which is 60 in our case -15 times that of the previous work [18]. Thus, the total time length of the phase-encoded sequence is $T=N \cdot \Delta t$. Here, the basic temporal element in the phase-encoded sequence is termed an "RF segment", which is a single-cycle or multi-cycle sine wave assembled from the input RF pulse (i.e., two anti-phase RF pulses assemble a single-cycle sine wave). The centre frequency of the phase-encoded sequence is determined by the frequency of the assembled sine waves, thus is equivalently given by $1 /(2 \Delta t)$. Assuming each RF segment constitutes $m$ pulses, then the length of the phase-encoded sequence would be $\mathrm{N} / \mathrm{m}$, together with an equivalent phase encoding speed of $1 /(m \Delta t)$. We note that the generated phase-encoded sequence has a bandwidth similar to the input RF pulse, which is subject to the Nyquist bandwidth, equivalent to half of the comb spacing $(48.9 \mathrm{GHz} / 2=24.45 \mathrm{GHz})$.

In a radar system, the phase encoded RF waveform is first amplified and then emitted by antennas, followed by reflection from the target, and finally collected and processed at the receiver. The range information of the target is carried on the delay of the received waveforms, which can be readily determined by calculating the autocorrelation function of the reflected phase-encoded RF signal given by 


$$
A C F=\int_{-\infty}^{+\infty} s(\tau) s(\tau+t) d \tau
$$

In practical systems this is calculated by performing a hardware autocorrelation extracted by an array of range gates. The range gates are composed of matched filters with their tap coefficients determined by the employed phase codes (Fig. 3). The function of the matched filters is to perform single-output autocorrelations for the received signal, and the range gate that has the highest output power denotes the resolved range of the target.

\section{EXPERIMENTAL RESULTS}

The experimental setup is shown in Figure 2. A CW laser is amplified and its polarization state adjusted to pump a nonlinear high Q factor ( $>1.5$ million) MRR, which featured a free spectral range of $\sim 0.4 \mathrm{~nm}$, or $48.9 \mathrm{GHz}$. As the detuning between the pump laser and the MRR was changed, dynamic parametric oscillation states corresponding to distinctive solutions of the Lugiato-Lefever equation were initiated [44]. We thus generated soliton crystal microcombs $[45,46]$, which were tightly packaged solitons circulating in the MRR as a result of a mode crossing (at $\sim 1552 \mathrm{~nm}$ in our case), and which resulted in the distinctive palm-like comb spectrum (Fig. 4).

Next, 60 lines of the microcomb were flattened ( $N=60$ in our case), using two stages of WaveShapers (Finisar 4000S) to acquire a high link gain and signal-to-noise ratio. This was achieved by pre-flattening the microcomb lines with the first WaveShaper such that the optical power distribution of the wavelength channels roughly matched with the desired channel weights. The second WaveShaper was employed for accurate comb shaping assisted by a feedback loop as well as to separate the wavelength channels into two parts (port 1 and port 2 of the Waveshaper) according to the polarity of the designed binary phase codes. The feedback loop was constructed by reading the optical spectrum with an optical spectrum analyser and comparing the power of the wavelengths with the designed weights to generate an error signal, which was then fed back into the second WaveShaper (WS2) to calibrate its loss until the error was below $0.2 \mathrm{~dB}$.

Here, we used a Gaussian pulse with a duration of $\Delta t=84 \mathrm{ps,} \mathrm{as} \mathrm{the} \mathrm{RF} \mathrm{fragment} f[t]$. Although we used an arbitrary waveform generator (Keysight, $65 \mathrm{GSa} / \mathrm{s}$ ) to generate the Gaussian pulse for the sake of simplicity, the arbitrary waveform generator is actually not a necessity and can be replaced with many other readily available approaches that are easier and cheaper [47, 48]. The input RF pulse was imprinted onto the comb lines, generating replicas across all the wavelength channels. The replicas then went through a $\sim 13 \mathrm{~km}$ long spool of standard single mode fibre (with a dispersion of $17 \mathrm{ps} / \mathrm{nm} / \mathrm{km}$ ) to progressively delay them, leading to a delay step of $\sim 84$ ps between the adjacent wavelength channels that matched with the duration of the RF pulse $\Delta t$. Finally, the wavelength channels were separated into two parts according to the designed phase codes and sent to a balanced photodetector (Finisar, $40 \mathrm{GHz}$ ) to achieve negative and positive replicas for the phase encoding.

Figure 5 shows the input Gaussian pulse and the flattened optical comb spectra measured at the output ports of the second Waveshaper (WS2). Each pair of wavelength channels from different ports can assemble a monocycle sine wave, thus with the 60 comb lines, 30 sine cycles can be achieved, with a total time length of $T=N \cdot \Delta t=60 \times 84 \mathrm{ps}=$ 5.04 ns.

By applying designed phase codes during the separation of the wavelength channels, the sine cycles could be $\pi$ phase shifted at desired times. The phase-encoded results are shown in Fig. 6 . The number of Gaussian pulses for each RF segment (denoted by $m$ ) was reconfigured from 6 to 2, corresponding to reconfigurable sequence lengths $(\mathrm{N} / \mathrm{m})$ ranging from 10 to 30 and phase coding speeds $(1 / \Delta t / m)$ ranging from 1.98 to $5.95 \mathrm{~Gb} / \mathrm{s}$. The employed phase codes were denoted both by the shaded areas and the stair waveforms (black solid line). This result shows that our photonic phase coder can offer a reconfigurable sequence length to address the performance tradeoffs between range resolution and system complexity. To acquire a large pulse compression ratio for a high resolution, the sequence length should be maximized, where the number of Gaussian pulses for each RF segment $(m)$ should be set as 2 . Further, to reduce the complexity and cost of the RF system (such as the number of range gates at the receiver), the sequence length could be reduced by either employing fewer wavelength channels or by increasing $m$.

The corresponding optical spectra (Fig. $6(\mathrm{a}, \mathrm{c}, \mathrm{e})$ ) were measured at the output of Waveshaper to show the positive and negative phase codes realized by changing the wavelength channels' output ports at the Waveshaper. The encoded RF waveforms (Fig. 6 (b, d, f)) clearly show the flipped phase of the RF segments at the time of negative phase codes, where the number of sine cycles was reconfigured as well, depending on the value of $m$. This result also shows that our approach is fully reconfigurable for different phase codes and encoding speeds. We note that higher encoding speeds can be achieved by reducing the duration of the RF fragment and the delay step $\Delta t$.

In this work we calculated the autocorrelation (Fig. 7) of the phase-encoded RF waveforms $s(t)$. As the sequence 
length varied from 10 to 30 , the full width at half-maximum (FWHM) of the compressed pulses varied from 0.52 to $0.17 \mathrm{~ns}$, which corresponds to a pulse suppression ratio ranging from 9.7 to 29.6. Meanwhile the peak-to-sidelobe ratio (PSR) also increased with the sequence length from 4.17 to $6.59 \mathrm{~dB}$. These results confirm that the pulse compression ratio of an RF phase-encoded signal is linearly related to its sequence length [49], and that this can be significantly enhanced with our approach by employing a larger number of wavelength channels of the microcomb.

Figure 8 shows calculated examples of the estimated outputs of the range gates [49] with different distances. Considering an example with a sequence length $N / m=30$, the delay of the matched filters would be $2 \Delta t=168 \mathrm{ps}$. The tap coefficients for the $l_{\text {th }}$ matched filter are $c[k-l]$, where $c[k], k=1,2, \ldots 30$, is the employed phase codes. The range resolution of the radar, which is the minimum distance between two resolvable targets, is determined by the delay step $(2 \Delta t)$ of the matched filters, which is given by $2 \Delta t \cdot c=5 \mathrm{~cm}$, where $c=3 \times 10^{8} \mathrm{~m} / \mathrm{s}$ is the speed of RF signals in air. If the distance between the target and radar is $R$, then the delay would be $2 R / c$. The range gates would have a maximum output at the $l_{\text {th }}$ range gate, $l=2 R /(c \cdot 2 \Delta t)$. We note here that this calculation only shows the basic connections between our phase encoder and radar systems' performances - practical radar systems are subject to more complicated tradeoffs involving capability versus performance.

We note that although random phase codes were used here as a proof of concept, the design of phase codes can be optimized to achieve a better detection performance of the radar system, which is an NP-hard problem and can be approximated and addressed with the methods in [50].

Further, the routes to further scale up the performance of our system are clear. The sequence length can be further increased by either employing microcombs that have a smaller FSR and thus more wavelength channels in the available optical band, or by introducing spatial-multiplexing techniques to boost the length of parallel channels. Moreover, the increasingly mature nonlinear platforms and recent advances in coherent soliton generation techniques, such as deterministic soliton generation, [51] battery-driven solitons [52], and laser cavity-solitons [53-56] enable microcombs to have an increasingly wide access and enhanced performance for engineering applications, including the phaseencoded signal generator reported here. Finally, other approaches towards delays and dispersion besides simple spools of fibre are available to realize compact latency free dispersive delays [57-62].

\section{CONCLUSION}

We demonstrate photonic RF phase encoding using an integrated micro-comb source. A single-cycle Gaussian pulse was multicast onto the comb wavelengths to assemble the desired phase-encoded RF waveform. A high pulse compression ratio of 29.6 and phase encoding speed of $5.95 \mathrm{~Gb} / \mathrm{s}$ were achieved, enabled by the use of 60 wavelengths generated by the microcomb. The sequence length was reconfigured by adjusting the length of each phase code, which led to a reconfigurable encoding speed. These results verify that our approach to high-speed RF phase encoding is competitive in terms of performance, with potentially lower cost and footprint.

\section{REFERENCES}

[1] P. Ghelfi, F. Laghezza, F. Scotti, G. Serafino, A. Capria, S. Pinna, D. Onori, C. Porzi, M. Scaffardi, A. Malacarne, V. Vercesi, E. Lazzeri, F. Berizzi, and A. Bogoni, “A fully photonics-based coherent radar system,” Nature, vol. 507, pp. 341, Mar. 2014.

[2] M. Skolnik, "Role of radar in microwaves," IEEE Transactions on Microwave Theory and Techniques, vol. 50, no. 3, pp. 625632. 2002.

[3] P. Ghelfi, F. Scotti, F. Laghezza, and A. Bogoni, "Photonic Generation of Phase-Modulated RF Signals for Pulse Compression Techniques in Coherent Radars," Journal of Lightwave Technology, vol. 30, no. 11, pp. 1638-1644, Jun. 2012.

[4] J. Capmany, and D. Novak, "Microwave photonics combines two worlds," Nat. Photonics, vol. 1, no. 6, pp. 319-330, Jun. 2007.

[5] J. P. Yao, "Microwave Photonics," J. Lightwave Technol., vol. 27, no. 1-4, pp. 314-335, Jan-Feb. 2009.

[6] D. Marpaung, J. Yao, and J. Capmany, "Integrated microwave photonics," Nature Photonics, vol. 13, no. 2, pp. 80-90, Feb. 2019.

[7] V. R. Supradeepa, C. M. Long, R. Wu, F. Ferdous, E. Hamidi, D. E. Leaird, and A. M. Weiner, "Comb-based radiofrequency photonic filters with rapid tunability and high selectivity," Nat. Photonics, vol. 6, no. 3, pp. 186-194, Mar. 2012.

[8] J. Wu, X. Xu, T. G. Nguyen, S. T. Chu, B. E. Little, R. Morandotti, A. Mitchell, and D. J. Moss, "RF Photonics: An Optical Microcombs' Perspective," IEEE Journal of Selected Topics in Quantum Electronics, vol. 24, no. 4, pp. 1-20. 2018.

[9] J. Azaña, “Ultrafast Analog All-Optical Signal Processors Based on Fiber-Grating Devices,” IEEE Photonics Journal, vol. 2, no. 3, pp. 359-386. 2010.

[10]Z. Jiang, C.-B. Huang, D. E. Leaird, and A. M. Weiner, “Optical arbitrary waveform processing of more than 100 spectral comb lines," Nature Photonics, vol. 1, pp. 463, Aug. 2007.

[11] S. T. Cundiff, and A. M. Weiner, "Optical arbitrary waveform generation,” Nature Photonics, vol. 4, pp. 760, Oct. 2010. 
[12] H. Chi, and J. Yao, "Photonic Generation of Phase-Coded Millimeter-Wave Signal Using a Polarization Modulator," IEEE Microwave and Wireless Components Letters, vol. 18, no. 5, pp. 371-373. 2008.

[13] Y. Zhang, and S. Pan, "Generation of phase-coded microwave signals using a polarization-modulator-based photonic microwave phase shifter," Optics Letters, vol. 38, no. 5, pp. 766-768, Mar. 2013.

[14]Z. Li, W. Li, H. Chi, X. Zhang, and J. Yao, "Photonic Generation of Phase-Coded Microwave Signal With Large Frequency Tunability," IEEE Photonics Technology Letters, vol. 23, no. 11, pp. 712-714. 2011.

[15] W. Liu, and J. Yao, "Photonic Generation of Microwave Waveforms Based on a Polarization Modulator in a Sagnac Loop," Journal of Lightwave Technology, vol. 32, no. 20, pp. 3637-3644, Oct. 2014.

[16] S. Zhu, Z. Shi, M. Li, N. H. Zhu, and W. Li, "Simultaneous frequency upconversion and phase coding of a radio-frequency signal for photonic radars," Optics Letters, vol. 43, no. 3, pp. 583-586, Feb. 2018.

[17] S. Zhu, M. Li, X. Wang, N. H. Zhu, Z. Z. Cao, and W. Li, "Photonic generation of background-free binary phase-coded microwave pulses," Optics Letters, vol. 44, no. 1, pp. 94-97, Jan. 2019.

[18] Y. Dai, and J. Yao, "Microwave pulse phase encoding using a photonic microwave delay-line filter," Optics Letters, vol. 32 , no. 24, pp. 3486-3488, Dec. 2007.

[19] T. J. Kippenberg, A. L. Gaeta, M. Lipson, and M. L. Gorodetsky, "Dissipative Kerr solitons in optical microresonators," Science, vol. 361, no. 6402, pp. 8083. 2018.

[20] D. J. Moss, R. Morandotti, A. L. Gaeta, and M. Lipson, "New CMOS-compatible platforms based on silicon nitride and Hydex for nonlinear optics," Nat. Photonics, vol. 7, no. 8, pp. 597-607, Aug. 2013.

[21] A. L. Gaeta, M. Lipson, and T. J. Kippenberg, "Photonic-chip-based frequency combs," Nature Photonics, vol. 13, no. 3, pp. 158-169, Mar. 2019.

[22] M.-G. Suh, and K. J. Vahala, "Soliton microcomb range measurement," Science, vol. 359, no. 6378, pp. 884-887. 2018.

[23] W. Liang, D. Eliyahu, V. S. Ilchenko, A. A. Savchenkov, A. B. Matsko, D. Seidel, and L. Maleki, "High spectral purity Kerr frequency comb radio frequency photonic oscillator," Nature communications, vol. 6, pp. 7957. 2015.

[24]D. T. Spencer, T. Drake, T. C. Briles, J. Stone, L. C. Sinclair, C. Fredrick, Q. Li, D. Westly, B. R. Ilic, and A. Bluestone, "An optical-frequency synthesizer using integrated photonics," Nature, vol. 557, no. 7703, pp. 81-85. 2018.

[25] X. Xu, J. Wu, T. G. Nguyen, M. Shoeiby, S. T. Chu, B. E. Little, R. Morandotti, A. Mitchell, and D. J. Moss, "Advanced RF and microwave functions based on an integrated optical frequency comb source," Opt. Express, vol. 26, no. 3, pp. 2569-2583, Feb 5. 2018.

[26] X. Xu, M. Tan, J. Wu, T. G. Nguyen, S. T. Chu, B. E. Little, R. Morandotti, A. Mitchell, and D. J. Moss, "Advanced Adaptive Photonic RF Filters with 80 Taps Based on an Integrated Optical Micro-Comb Source," Journal of Lightwave Technology, vol. 37, no. 4, pp. 1288-1295, Feb. 2019.

[27] X. X. Xue, Y. Xuan, H. J. Kim, J. Wang, D. E. Leaird, M. H. Qi, and A. M. Weiner, "Programmable Single-Bandpass Photonic RF Filter Based on Kerr Comb from a Microring," J. Lightwave Technol., vol. 32, no. 20, pp. 3557-3565, Oct 15. 2014.

[28] X. Xu, J. Wu, M. Shoeiby, T. G. Nguyen, S. T. Chu, B. E. Little, R. Morandotti, A. Mitchell, and D. J. Moss, "Reconfigurable broadband microwave photonic intensity differentiator based on an integrated optical frequency comb source," APL Photonics, vol. 2, no. 9, 096104 Sep. 2017.

[29] X. Xu, J. Wu, T. G. Nguyen, T. Moein, S. T. Chu, B. E. Little, R. Morandotti, A. Mitchell, and D. J. Moss, "Photonic microwave true time delays for phased array antennas using a $49 \mathrm{GHz}$ FSR integrated optical micro-comb source," Photonics Res, vol. 6, no. 5, pp. B30-B36, May 1. 2018.

[30] X. Xue, Y. Xuan, C. Bao, S. Li, X. Zheng, B. Zhou, M. Qi, and A. M. Weiner, "Microcomb-Based True-Time-Delay Network for Microwave Beamforming with Arbitrary Beam Pattern Control," Journal of Lightwave Technology, vol. 36, no. 12, pp. 2312-2321, Jun. 2018.

[31] X. Xu, M. Tan, J. Wu, T. G. Nguyen, S. T. Chu, B. E. Little, R. Morandotti, A. Mitchell, and D. J. Moss, "High performance RF filters via bandwidth scaling with Kerr micro-combs," APL Photonics, vol. 4, no. 2, 026102. 2019.

[32] X. Xu, J. Wu, T. G. Nguyen, S. Chu, B. Little, A. Mitchell, R. Morandotti, and D. J. Moss, "Broadband RF Channelizer based on an Integrated Optical Frequency Kerr Comb Source," Journal of Lightwave Technology, vol. 36, no. 19, pp. 4519-4526, 2018.

[33] M.Tan, X.Xu, B.Corcoran, J.Wu, A. Boes, T. G. Nguyen, S.T. Chu, B.E. Little, R. Morandotti, A.Mitchell, and D.J. Moss, "Broadband microwave and RF photonic fractional Hilbert transformer based on a 50GHz integrated Kerr micro-comb", Journal of Lightwave Technology, vol. 37, no. 24, 6097 - 6104, 2019. DOI:10.1109/JLT.2019.2946606.

[34] T.G. Nguyen, M.Shoeiby, S.T. Chu, B.E. Little, R. Morandotti, A. Mitchell, and D. J. Moss, "Integrated frequency comb source based Hilbert transformer for wideband microwave photonic phase analysis", Optics Express, Vol. 23, no. 17, 22087 (2015). DOI:10.1364/OE.23.022087

[35] X. Xu, M. Tan, J.Wu, R.Morandotti, A.Mitchell, and D. J. Moss, "Microcomb-based photonic RF signal processing", IEEE Photonics Technology Letters, Vol. 31 no. 23, pp.1854-1857 (2019). DOI: 10.1109/LPT.2019.2940497

[36]X.Xu, J.Wu, Mengxi Tan, T.G. Nguyen, S.T. Chu, B. E. Little, R.Morandotti, A.Mitchell, and D. J. Moss, "Broadband microwave frequency conversion based on an integrated optical micro-comb source", Journal of Lightwave Technology, Vol. 38, no. 2, pp.332-338 (2020). DOI: 10.1109/JLT.2019.2930466

[37] A. Pasquazi; M.Peccianti; B. Little; S.T. Chu; D. J. Moss; R. Morandotti, "Stable, dual mode, high repetition rate mode-locked laser based on a microring resonator", Optics Express vol. 20, no. 24, 27355 - 27362, 2012. DOI: 10.1364/OE.20.027355 
[38]M.Tan et al., "Broadband microwave and RF fractional differentiator using photonics", IEEE Transactions on Circuits and Systems: Express Briefs, Early Access 37 (2020). DOI:10.1109/TCSII.2020.2965158

[39]B. Corcoran et al., "Ultra-dense optical data transmission over standard fiber with a single chip source", Nature Communications 11 Article number: 2568, May 22 (2020). DOI:10.1038/s41467-020-16265-x.

[40] M. Tan et al., "Photonic RF arbitrary waveform generator based on a soliton crystal micro-comb source", Journal of Lightwave Technology Early Access $\underline{\mathbf{3 8}}$ (2020). DOI:10.1109/JLT.2020.3009655.

[41] X. Xu et al., "Broadband photonic radio frequency channelizer with 90 channels based on a soliton crystal microcomb", Journal of Lightwave Technology, vol. 38, no. 2, pp. 332-338, Jan. 15, (2020). DOI: 10.1109/JLT.2019.2930466.

[42] X.Xu et al., "Photonic RF and microwave integrator with soliton crystal microcombs", IEEE Transactions on Circuits and Systems: Express Briefs, Early Access 67 May 19 (2020). DOI:10.1109/TCSII.2020.2995682

[43]X. Xu et al., "Photonic perceptron based on a Kerr microcomb for scalable high speed optical neural networks", Laser and Photonics Reviews 14 (8) 2000070 (2020). DOI:10.1002/lpor.202000070.

[44] A. Pasquazi, M. Peccianti, L. Razzari, D. J. Moss, S. Coen, M. Erkintalo, Y. K. Chembo, T. Hansson, S. Wabnitz, P. Del'Haye, X. X. Xue, A. M. Weiner, and R. Morandotti, "Micro-combs: A novel generation of optical sources," Physics Reports, vol. 729, pp. 1-81, Jan 27. 2018.

[45] D. C. Cole, E. S. Lamb, P. Del'Haye, S. A. Diddams, and S. B. Papp, "Soliton crystals in Kerr resonators," Nat. Photonics, vol. 11, no. 10, pp. 671-676, Oct. 2017.

[46] W. Q. Wang, Z. Z. Lu, W. F. Zhang, S. T. Chu, B. E. Little, L. R. Wang, X. P. Xie, M. L. Liu, Q. H. Yang, L. Wang, J. G. Zhao, G. X. Wang, Q. B. Sun, Y. S. Liu, Y. S. Wang, and W. Zhao, "Robust soliton crystals in a thermally controlled microresonator," Opt. Lett., vol. 43, no. 9, pp. 2002-2005, May 1. 2018.

[47] W. Chiming, and N. K. Dutta, "High-repetition-rate optical pulse generation using a rational harmonic mode-locked fiber laser," IEEE Journal of Quantum Electronics, vol. 36, no. 2, pp. 145-150. 2000.

[48] H. Jeongwoo, and N. Cam, "A new ultra-wideband, ultra-short monocycle pulse generator with reduced ringing," IEEE Microwave and Wireless Components Letters, vol. 12, no. 6, pp. 206-208. 2002.

[49] N. Levanon, and B. Getz, "Comparison between linear FM and phase-coded CW radars," IEE Proceedings - Radar, Sonar and Navigation, vol. 141, no. 4, pp. 230-240. 1994.

[50] A. D. Maio, S. D. Nicola, Y. Huang, Z. Luo, and S. Zhang, "Design of Phase Codes for Radar Performance Optimization with a Similarity Constraint," IEEE Transactions on Signal Processing, vol. 57, no. 2, pp. 610-621. 2009.

[51] H. Zhou, Y. Geng, W. Cui, S.-W. Huang, Q. Zhou, K. Qiu, and C. Wei Wong, "Soliton bursts and deterministic dissipative Kerr soliton generation in auxiliary-assisted microcavities," Light: Science \& Applications, vol. 8, no. 1, pp. 50, 2019.

[52]B. Stern, X. Ji, Y. Okawachi, A. L. Gaeta, and M. Lipson, "Battery-operated integrated frequency comb generator," Nature, vol. 562, no. 7727, pp. 401. 2018.

[53]H. Bao et al., "Laser cavity-soliton microcombs," Nature Photonics, vol. 13, no. 6, pp. 384-389, Jun. 2019.

[54] H. Bao et al., "Turing patterns in a fibre laser with a nested micro-resonator: robust and controllable micro-comb generation", Physical Review Research $\underline{\mathbf{2}}$ (2), 023395 (2020).

[55]L. D. Lauro et al., "Parametric control of thermal self-pulsation in micro-cavities," Opt. Lett. vol. 42, no. 17, pp. 3407-3410, Aug. 2017.

[56]H. Bao et al., "Type-II micro-comb generation in a filter-driven four wave mixing laser," Photonics Research, vol. 6, no. 5, pp. B67-B73, May 2018.

[57]D.J. Moss et al., "Tunable dispersion and dispersion slope compensators for 10Gb/s using all-pass multicavity etalons", IEEE Photonics Technology Letters, vol. 15, no. (5), 730-732 (2003). DOI: 10.1109/LPT.2003.809921.

[58]D.J. Moss et al. "Multichannel tunable dispersion compensation using all-pass multicavity etalons", paper TuT2 Optical Fiber Communications Conference, Anaheim (2002). Postconference Technical Digest (IEEE Cat. No.02CH37339). Opt Soc. America. Part vol.1, 2002, pp. 132-3. Washington, DC, USA.

[59]D.J. Moss et al., "Tunable dispersion compensation at $10 \mathrm{~Gb} / \mathrm{s}$ and $40 \mathrm{~Gb} / \mathrm{s}$ using multicavity all-pass etalons", Optical Fiber Communications Conference (OFC) paper TuD1, page 162, Atlanta, GA, March (2003). Post-conference Digest (IEEE Cat. No.03CH37403). Opt. Soc. America. Part vol.1, 2003, pp. 162-3. Washington, DC, USA.

[60] L. Lunardi et al.,"Tunable dispersion compensators based on multi-cavity all-pass etalons for 40Gb/s systems", Journal of Lightwave Technology, vol. 20, no. 12, 2136 (2002). DOI: 10.1109/JLT.2002.806768.

[61]L.M. Lunardi et al., "An Etalon-Based Tunable Dispersion Compensator Device for 40-Gbit/s Applications", European Conf. on Optical Communications, Paper 5.4.6 Copenhagen, Sept. (2002). vol. 2, 2002, Piscataway, NJ, USA. Print ISBN: 8790974-63-8.

[62] M Shokooh-Saremi, VG Ta'Eed, NJ Baker, ICM Littler, DJ Moss, et al., "High-performance Bragg gratings in chalcogenide rib waveguides written with a modified Sagnac interferometer", JOSA B Vol. 23, No. 7, pp.1323-1331 (2006). 

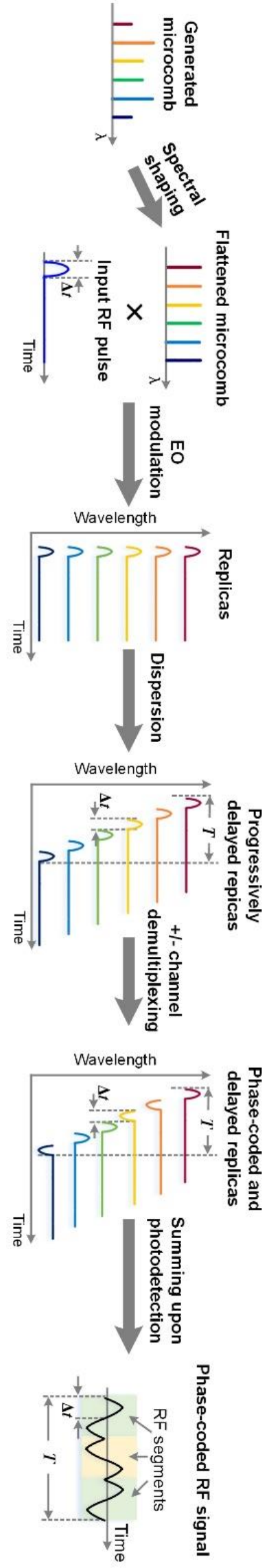

Fig. 1. Illustration of the operation of the photonic RF phase encoder. 


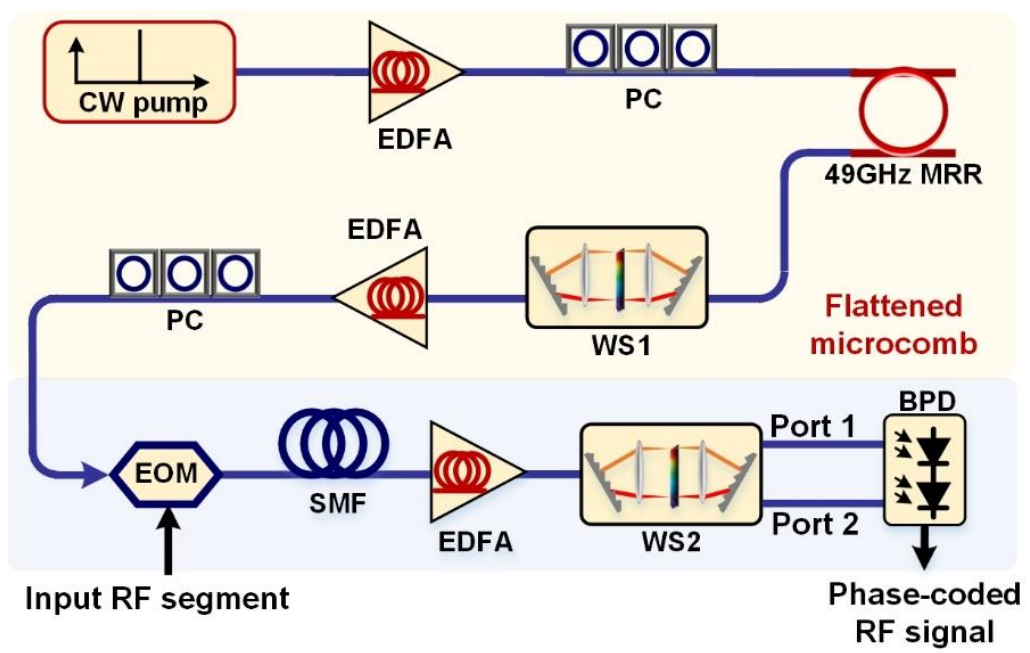

Fig. 2. Schematic diagram of photonic RF phase encoding based on an integrated optical micro-comb source. EDFA: erbium-doped fibre amplifier. PC: polarization controller. MRR: micro-ring resonator. WS: WaveShaper. EOM: Mach-Zehnder modulator. SMF: Single mode fibre. BPD: balanced photodetector.

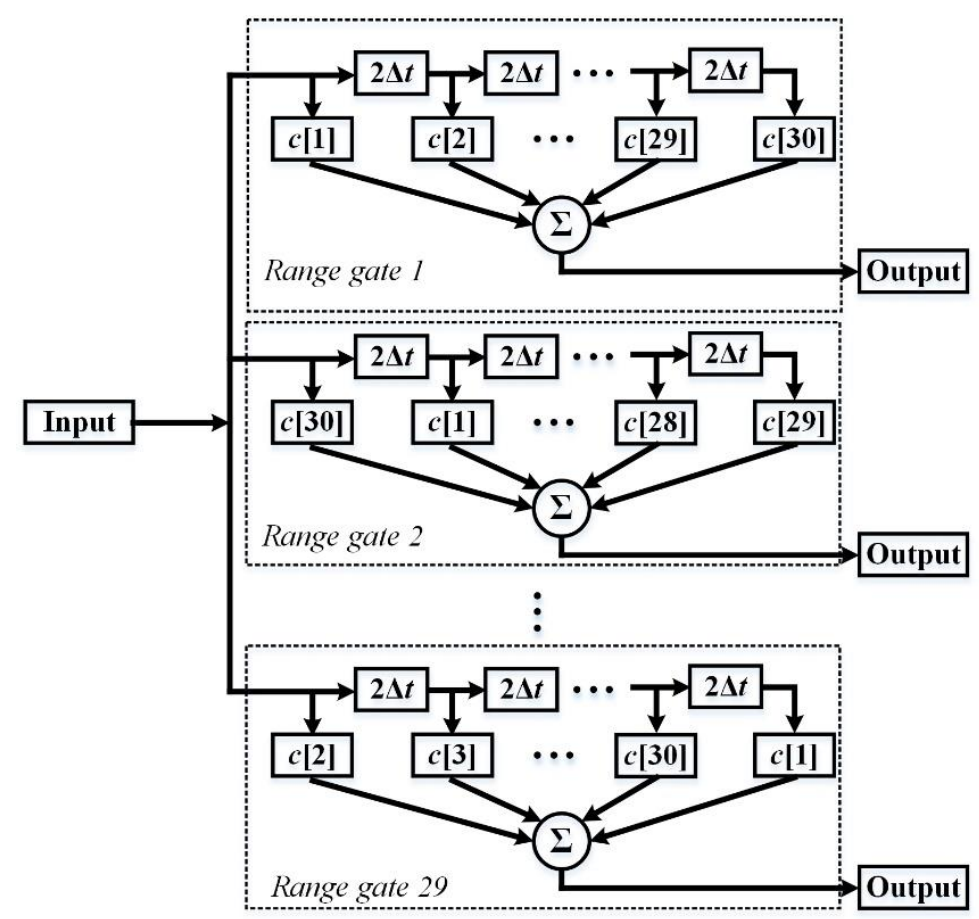

Fig. 3. Structure of the range gates at the radar systems' receiver. 


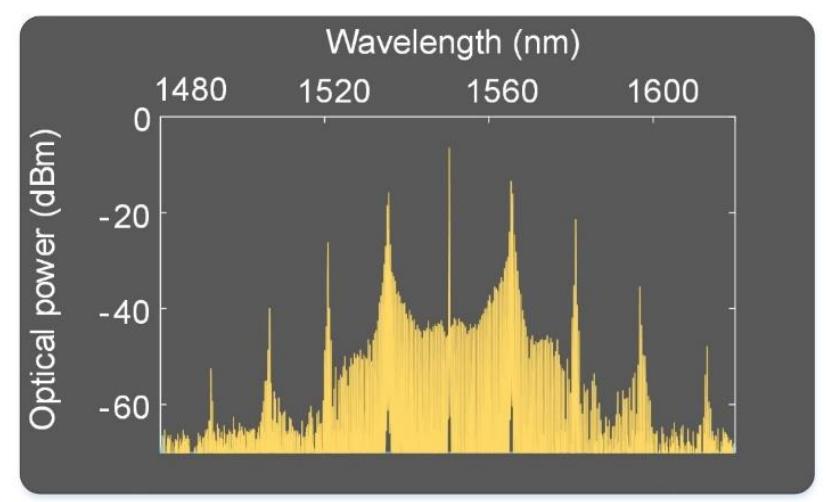

Fig. 4. Soliton crystal microcomb.
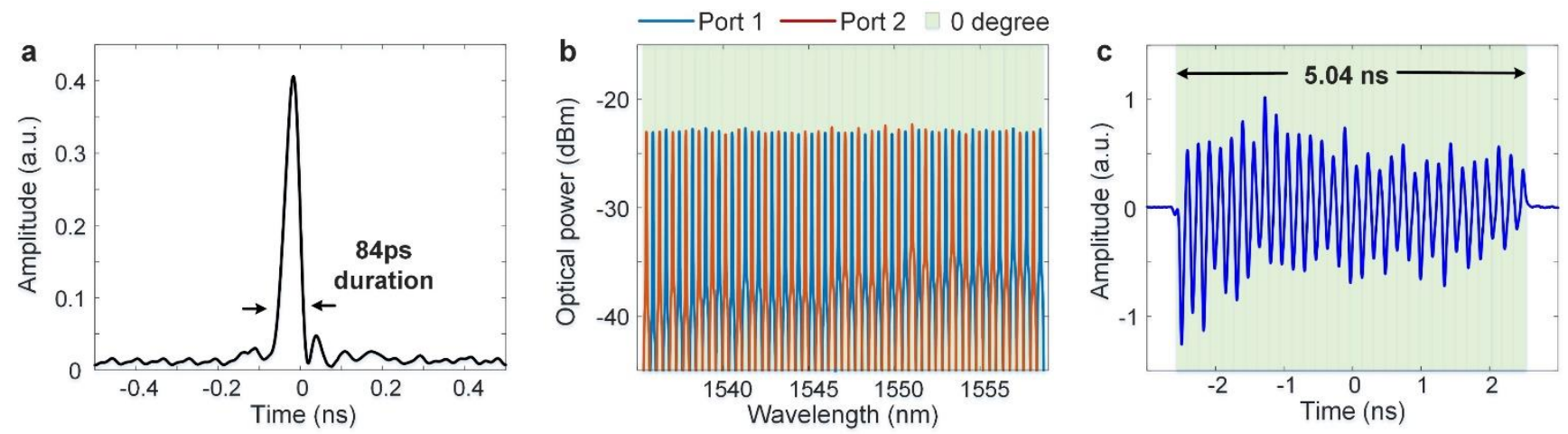

Fig. 5. (a) The input Gaussian pulse. (b) The optical spectra of the flattened microcomb at different ports of the Waveshaper. (c) Assembled $\mathrm{RF}$ signal with 30 cosine cycles. 

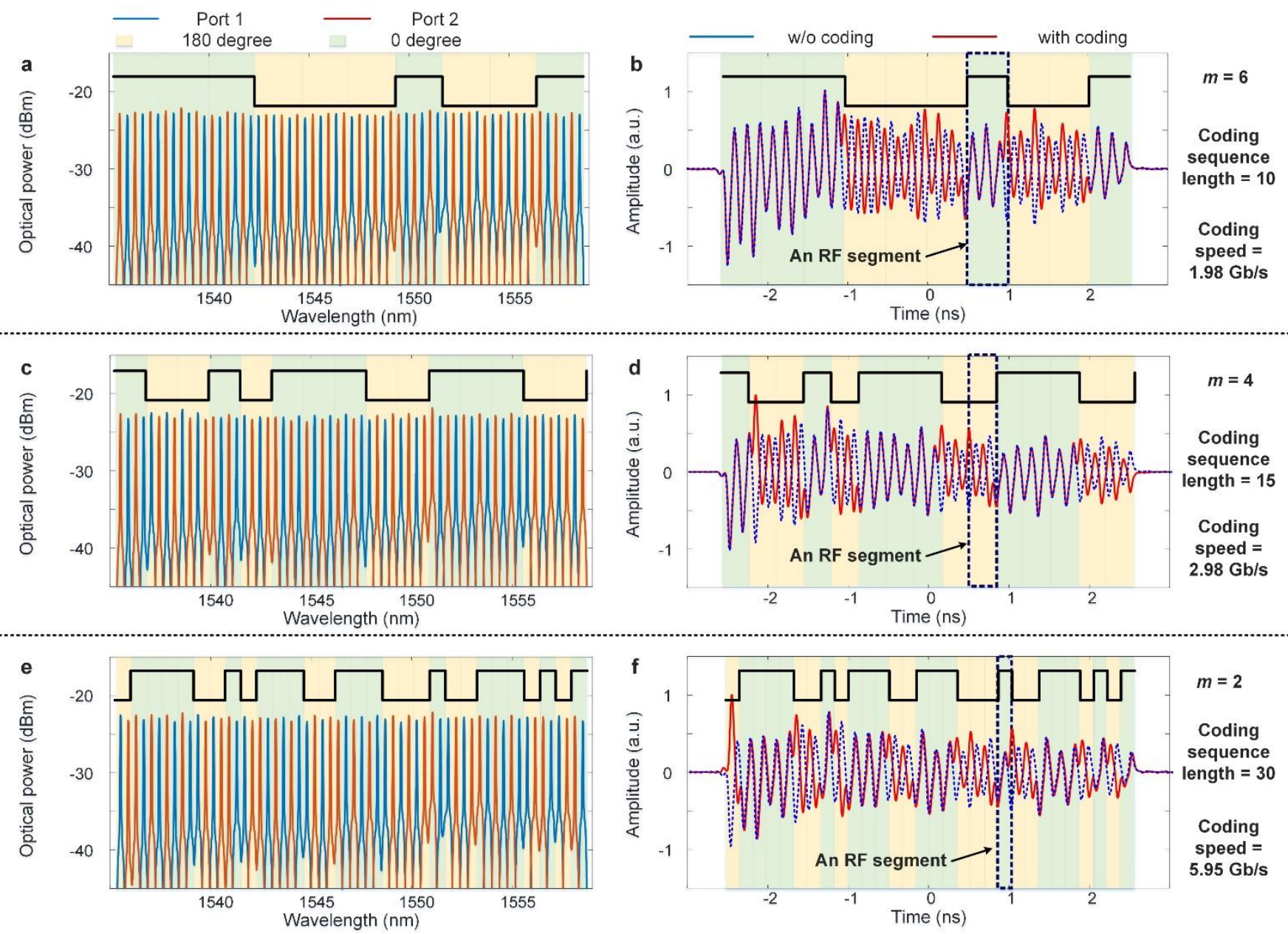

Fig. 6. (a, c, e) The optical spectra of the flattened microcomb at different ports of the Waveshaper with different phase codes and sequence length. (b, d, f) The assembled phase-encoded RF waveform.
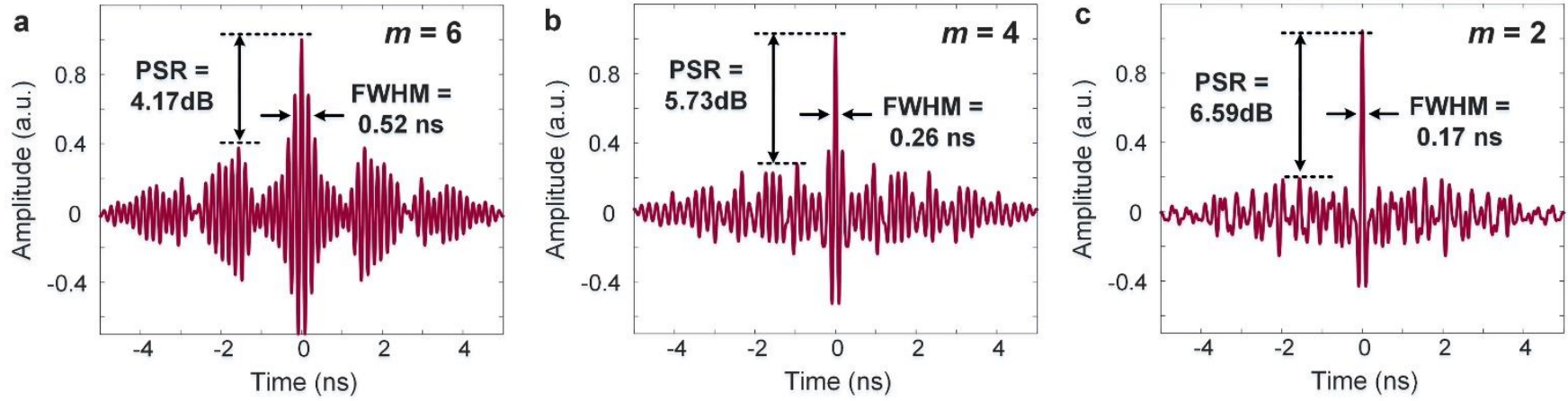

Fig. 7. The calculated autocorrelation of the phase-encoded RF waveforms for (a) $m=6$, (b) $m=4$, and (c) $m=2$. 

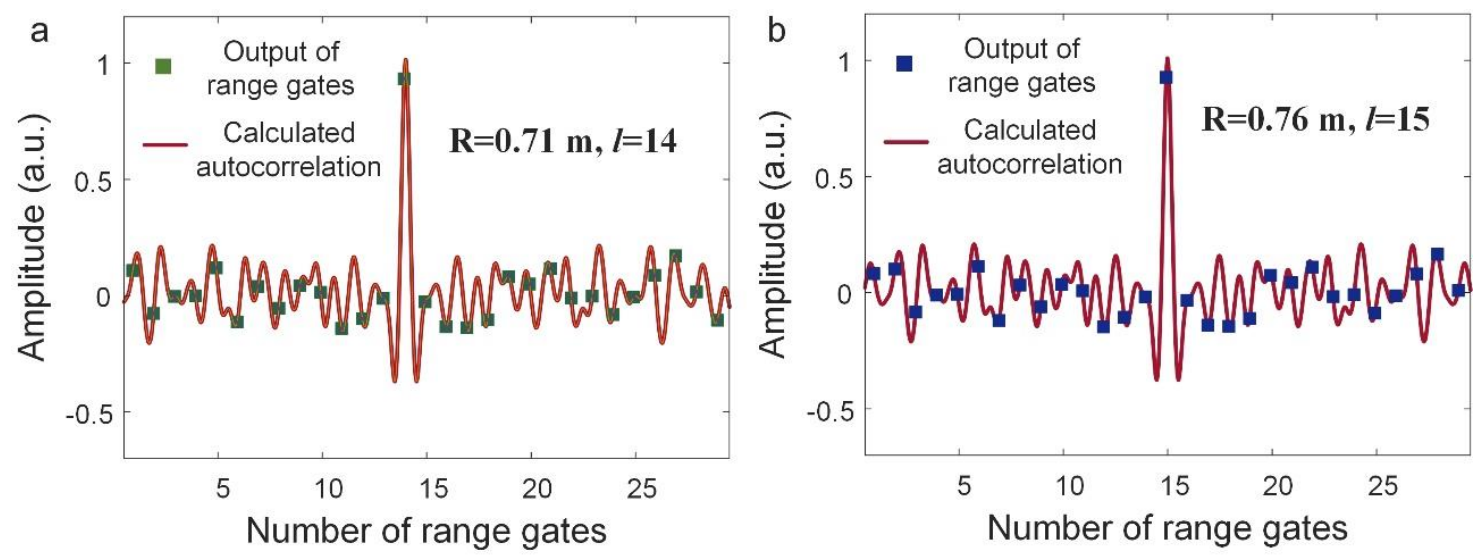

Fig. 8. Calculated outputs of the range gates for (a) $R=0.71 \mathrm{~m}$ and (b) $R=0.76 \mathrm{~m}$. 